\title{
Design of a Wearable Skin Stretch Cutaneous Device for the Upper Limb
}

\author{
Francesco Chinello ${ }^{1,2}$, Claudio Pacchierotti ${ }^{2}$, Nikos G. Tsagarakis ${ }^{2}$, and Domenico Prattichizzo ${ }^{1,2}$
}

\begin{abstract}
This paper presents a novel cutaneous device capable of providing independent skin stretches at the palmar, dorsal, ulnar, and radial sides of the arm. It consists of a lightweight bracelet with four servo motors. Each motor actuates a cylindrical shaped end-effector that is able to rotate, generating skin stretch stimuli. To understand how to control and wear the device on the forearm to evoke the most effective cutaneous sensations, we carried out perceptual experiments evaluating its absolute and differential thresholds. Finally, we carried out an experiment of haptic navigation to assess the effectiveness of our device as a navigation feedback system to guide a desired rotation and translation of the forearm. Results demonstrate an average rotation and translation error of $1.87^{\circ}$ and $2.84 \mathrm{~mm}$, respectively. Moreover, all the subjects found our device easy to wear and comfortable. Nine out of ten found it effective in transmitting navigation information to the forearm.
\end{abstract}

\section{INTRODUCTION}

The richness of information cutaneous receptors are able to detect, together with their broad distribution throughout the body, makes the skin a perfect channel to communicate with the human user [1]. Moreover, cutaneous feedback provides an effective and elegant way to simplify the design of this type of haptic interfaces: cutaneous receptors' very low activation thresholds [2] enable researchers to design small, lightweight and inexpensive cutaneous haptic interfaces [3], [4], [5]. Finally, cutaneous feedback has been also proven to play a key role in enhancing the performance and effectiveness of teleoperation and immersive systems [6], [7], [8], [9], [10].

An example of a cutaneous device exploiting these capabilities is the one presented by Prattichizzo et al. [5], developed to provide contact deformations stimuli at the fingertip. The device weights only $35 \mathrm{~g}$, and it is composed of two platforms: one is located on the back of the finger, supporting three small DC motors, and the other is in contact with the volar surface of the fingertip. The motors shorten and lengthen three cables to move the platform toward the user's fingertip and re-angle it to simulate contacts with arbitrarily oriented surfaces. The direction and amount of the force reflected to the user is changed by properly controlling the cable lengths. A similar device was also used to display remote tactile experiences [11] and unobtrusively interact with virtual and augmented environments [10]. Similarly, Solazzi et al. [12] developed a 3-DoF wearable cutaneous display to render virtual slanted surfaces. Four motors are

This research has received funding from the European Union Seventh Framework Programme FP7/2007-2013 under grant agreement $n^{\circ} 601165$ of the project "WEARHAP - WEARable HAPtics for humans and robots" and under grant agreement $\mathrm{n}^{\circ} 643644$ of the project "ACANTO: A CyberphysicAl social NeTwOrk using robot friends".

${ }^{1}$ Department of Information Engineering and Mathematics, University of Siena, Via Roma 56, 53100 Siena, Italy.

2 Department of Advanced Robotics, Istituto Italiano di Tecnologia, Via Morego 30, 16163 Genova, Italy.

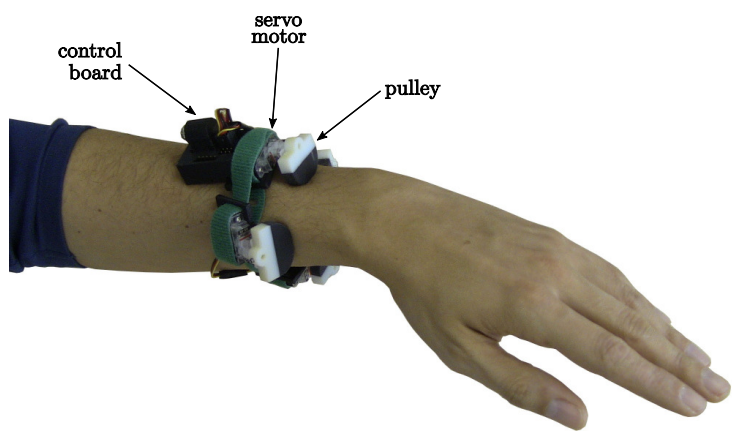

(a) Side view

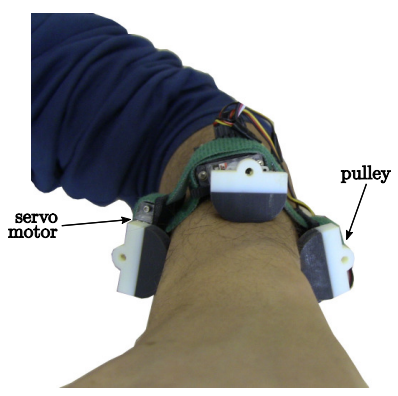

(b) Front view

Fig. 1. A prototype of the cutaneous device worn by the user. Four cylindrical rotating end-effectors provide the user with independent skin stretches at the palmar, dorsal, ulnar, and radial sides of the arm.

placed on the forearm and two cables for each actuated finger transmit the motor torque to the fingertips. In addition to the above mentioned type of cutaneous devices, there is also a growing interest in vibrotactile cutaneous feedback. Vibrations have been in fact successfully employed to provide navigation information and contact acceleration feedback in many scenarios [13], [14], [15]. Erp et al. [13], for example, explored the possibility of presenting navigation information through a vibrating waist belt. Results indicate the usefulness of vibrotactile cues for navigation purposes as well as for situational awareness in multi-tasks environments. A third type of devices providing cutaneous stimuli are the ones able to apply lateral stretches to the user's skin. They exploit the high sensitivity of human skin to tangential stretches and can provide the user with directional information. Conversely to vibration, skin stretch stimuli can be used to activate both slow-acting (SA) and fast acting (FA) mechanoreceptors. Gleeson et al. [16], for example, introduced a two-degreeof-freedom (2-DoF) cutaneous device that laterally stretches the skin of the fingertip using a $7 \mathrm{~mm}$ hemispherical tactor. Its two RC servo motors and compliant flexure stage can move the tactor along any path in the plane of the fingerpad. The device is capable of rendering $1 \mathrm{~mm}$ of displacement 


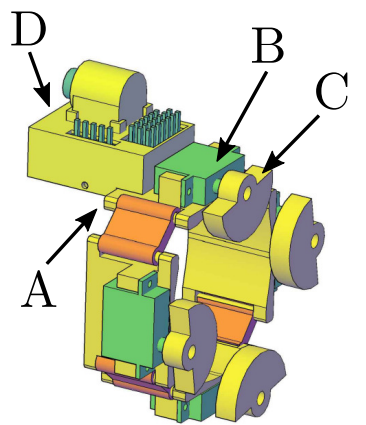

(a) CAD design

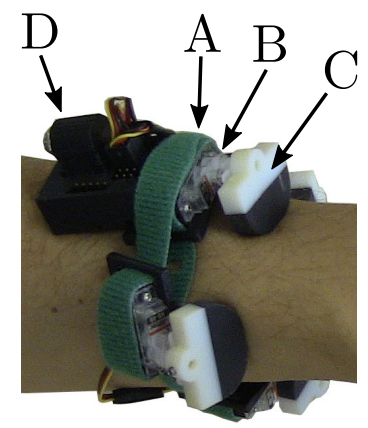

(b) Device being worn.
Fig. 2. The proposed skin stretch device. The device is composed of four static platforms (A) that house four servomotors (B), and four output pulleys (C) able to apply the requested stimuli to the skin. The four static platforms are connected by fabric straps to form a lightweight bracelet.

at arbitrary orientations within a plane, with a rate of 5 $\mathrm{mm} / \mathrm{s}$. The device has been also used to guide a human user navigating an unknown space [17]. Minamizawa et al. [3] developed a wearable cutaneous device able to render the weight of virtual objects by providing, at the same time, cutaneous stimuli tangential and normal to the finger pulp. It consists of two motors that move a belt that is in contact with the user's fingertip. When the motors spin in opposite directions, the belt presses into the user's fingertip, while when the motors spin in the same direction, the belt applies a tangential force to the skin.

Although the fingertip is often considered a privileged channel to convey cutaneous stimuli, in the literature it is easy to find cutaneous devices providing forces in other areas of the body. In this respect, the forearm has been often chosen as an effective location to provide feedback to the hand without impairing the hand itself [18], [19], [20], [21]. Bark et al. [22], for example, presented a wearable haptic feedback device able to impart rotational skin stretch to the hairy skin of the forearm. Subjects were able to use feedback from the device to control the positioning of a virtual object within \pm 6.5 degrees of the total range of motion. Nakamura et al. [23] presented a device to control hanger reflex generation at the wrist. The device is composed of a round cast and two urethane bands shaped to press upon the wrist when the device rotates. Kuniyasu et al. [24] developed a wearable haptic device that guides the motion of the arm using two rotating plates placed at the palmar and dorsal sides of the forearm. Stanley and Kuchenbecker [25] evaluated ten different forms of tactile feedback for wrist rotation guidance. The devices are capable of tapping, dragging across, squeezing, twisting, or vibrating against the user's wrist. Results show that directional responses are fastest when direction is conveyed through the location of the tactile stimulus or steady lateral skin stretch. The best performance was achieved by a device that repeatedly taps on the subject's wrist on the side toward which they should turn. More recently, Yem et al. [26] presented a wearable outer-covering haptic display for hand motion. It is composed of two spherical end-effectors that provide guidance information about the rotation and translation of the wrist.
In this work we present a novel cutaneous haptic device able to provide navigation cues through lateral skin stretch haptic feedback, shown in Figs. 1 and 2. Four cylindrical rotating end-effectors, placed on the forearm of the human user, can generate independent skin stretches at the palmar, dorsal, ulnar, and radial sides of the arm. When all the end-effectors rotate in the same direction, the cutaneous device is able to provide cutaneous cues about a desired pronation/supination of the forearm (see Figs. 3a and 3b). On the other hand, when two opposite end-effectors rotate in different directions, the cutaneous device is able to provide cutaneous cues about a desired translation of the forearm (see Figs. 3c and 3d). With respect to other systems presented in the literature, this device is extremely wearable and unobstrusive. Moreover, it can provide information about both the rotation and translation of the wrist.

\section{THE SKIN STRETCH HAPTIC DEVICE}

The proposed skin stretch cutaneous device is sketched in Fig. 2a, while a prototype is shown in Figs. 1 and $2 b$. The device is composed of four static platforms (labeled as "A" in Fig. 2), that accommodate four servomotors (B), and four cylindrical shaped end-effectors (C), that apply the requested stimuli to the skin. The four static platforms are connected by fabric straps, forming a lightweight bracelet. The actuators used for the prototype are HS-40 servomotors (Hitec, Republic of Korea). The maximum stall torque of one motors is $0.6 \mathrm{~kg} \cdot \mathrm{cm}$ at $4.8 \mathrm{~V}$. The pulley end-effectors and the mechanical support are made with a special type of acrylonitrile butadiene styrene, called ABSPlus (Stratasys, USA). The end-effectors are then covered with a rubber layer to improve grip and reduce slipping while in contact with the skin. The device is powered at $5 \mathrm{~V}$ by an external adapter. When all the end-effectors rotate in the same direction, the cutaneous device is able to provide cues about a desired pronation/supination of the forearm (see Figs. 3a and 3b). On the other hand, when two opposite end-effectors rotate in different directions, the cutaneous device is able to provide cues about a desired translation of the forearm (see Figs. 3c and $3 d)$.

\section{A. Kinematics of one module}

If no slip occurs between the mobile end-effector and the user's skin, the displacement $\overline{\Delta S}$ of the end-effector can be considered as the skin stretch provided by our cutaneous device onto the user's skin. This displacement can be evaluated as

$$
\overline{\Delta S}=\Delta \alpha \cdot r,
$$

where $r=20 \mathrm{~mm}$ is the radius of the end-effector (see Fig. 4) and $\Delta \alpha$ is the commanded angle variation expressed in radians.

\section{Perceptual thresholds}

To understand (i) how to correctly modulate the reference input of the device, (ii) where to locate it, and (iii) how to wear it to evoke the most effective cutaneous sensations, we 


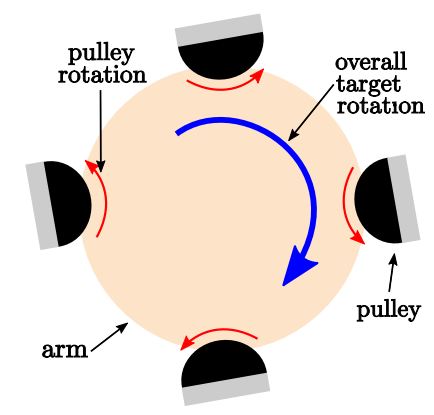

(a) Guidance for clockwise rotation (pronation)

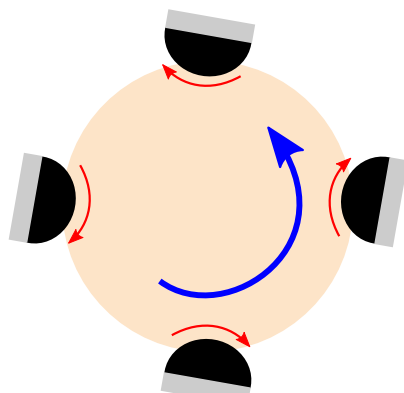

(b) Guidance for counter-clockwise rotation (supination)

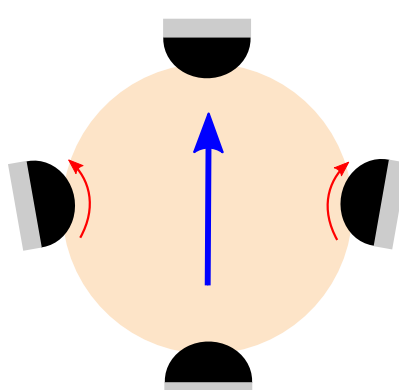

(c) Vertical motion of the wrist (dorsal motion)

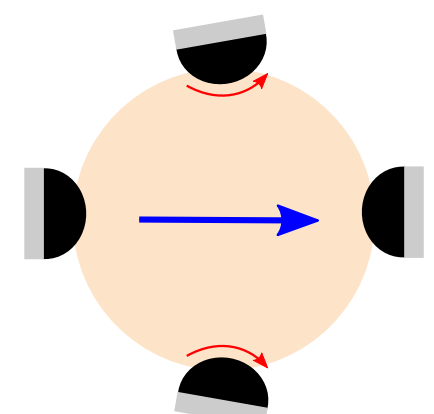

(d) Horizontal motion of the wrist (ulnar motion)

Fig. 3. Working principle. When all the end-effectors rotate in the same direction, the cutaneous device is able to provide cutaneous cues about a desired pronation/supination of the forearm (see (a) and (b)). On the other hand, when two opposite end-effectors rotate in different directions, the cutaneous device is able to provide cutaneous cues about a desired translation of the forearm (see (c) and (d)).

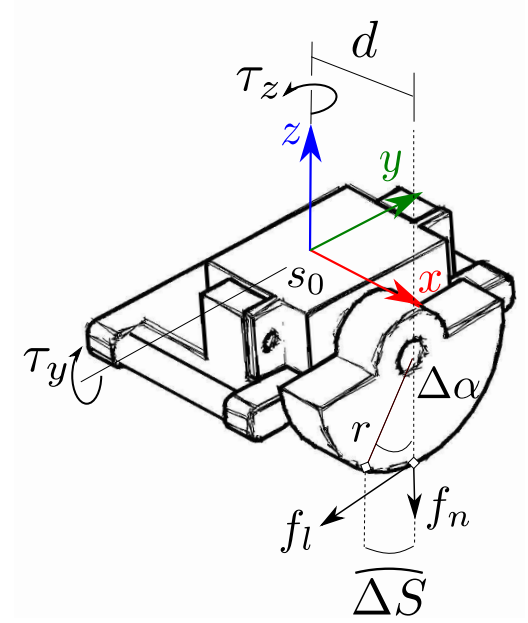

Fig. 4. Kinematic scheme of the skin stretch device.

carried out two preliminary experiments aiming at evaluating its absolute and differential thresholds.

We decided to test these two metrics in 8 different working conditions, changing the position of the device along the arm and the normal force exerted on the skin (i.e., how tight it was). We tested the metrics with the device worn either $4 \mathrm{~cm}$ proximal to the lunate bone (see Fig. $5 b$ ) and $10 \mathrm{~cm}$ proximal to the lunate bone (see Fig. 5c). Moreover, we tested the metrics when the end-effector applies a normal force $f_{n}$ to the skin of $2 \mathrm{~N}, 4 \mathrm{~N}, 6 \mathrm{~N}$, and $8 \mathrm{~N}$ (see Fig. 4).

The experimental setup is shown in Fig. 5. We modified the device to consider only the end-effector placed on the dorsal side of the arm. A six-axis force/torque sensor (ATI Nano 25, ATI Industrial Automation, USA) was mounted between the static platform (the one labeled as "A" in Fig. 2) and an external structure in order to measure the interaction forces between the device and the skin, associating the normal component of these forces to how tight the device was fastened to the user's arm. A screw enabled the experimenter to easily modulate the force exerted by the device. The sensor was also used to detect any slippage of the end-effector on the skin through the monitoring of the lateral force $f_{l}$. A white cardboard prevented the subjects from seeing the device.
The force $f$ applied by the device to the skin can be evaluated as

$$
\boldsymbol{f}=\left[\begin{array}{l}
f_{l} \\
f_{n}
\end{array}\right]=\left[\begin{array}{c}
-\frac{\tau_{z}}{d} \\
-\frac{\tau_{y}}{d}
\end{array}\right],
$$

where $\tau_{y}$ and $\tau_{z}$ are the torques registered by the ATI sensor w.r.t. the reference frame $s_{0}=\langle x, y, z\rangle$ placed at the base of the sensor, $f_{l}$ and $f_{n}$ are the lateral and normal forces exerted by the mobile end-effector to the skin, respectively, and $d=30 \mathrm{~mm}$ is the distance between the end-effector and the origin of $s_{0}$ (see Fig. 4).

\section{A. Absolute threshold}

The absolute threshold can be defined as the "smallest amount of stimulus energy necessary to produce a sensation" [27], and provides information about the smallest displacement we need to generate with the device to produce a skin stretch sensation perceivable by the human user.

Ten participants took part in the experiment, including two women and eight men. Six of them had previous experience with haptic interfaces. None of the participants reported any deficiencies in their visual or haptic perception abilities, and all of them were right-hand dominant.

We evaluated the absolute threshold using the simple updown method [28]. We used a step-size $\Delta \alpha=1^{\circ}$, that corresponded to a stretch of $0.35 \mathrm{~mm}$ on the skin (see eq. (1)). We considered the task completed when six reversals occurred. Subjects were required to wear the cutaneous device as shown in Fig. 5 and tell the experimenter when they felt the stimulus, i.e., when they felt a stretch on the skin.

Each participant performed sixteen trials of the simple up-down procedure, with two repetitions for each of the four force values considered ( $2 \mathrm{~N}, 4 \mathrm{~N}, 6 \mathrm{~N}, 8 \mathrm{~N}$ ) and each of the two position considered $(4 \mathrm{~cm}$ and $10 \mathrm{~cm}$ proximal to the lunate bone). Fig. 6 shows the absolute thresholds registered in the eight working conditions. By examining the lateral forces registered through the ATI F/T sensor it was verified that no slippage effect took place during these trials. As expected, when the device is well tight and, therefore, the mobile end-effector exert a higher pressure on the user's skin, the absolute threshold is lower. Moreover, it seems that the 


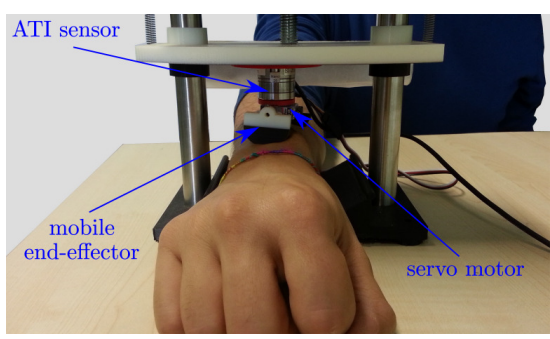

(a) Front view

(4 $\mathrm{cm}$ proximal to the lunate bone)

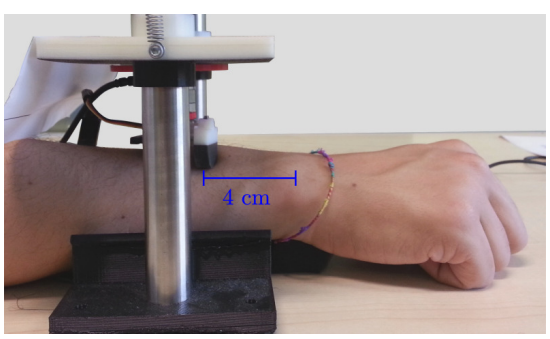

(b) Side view

(4 $\mathrm{cm}$ proximal to the lunate bone)

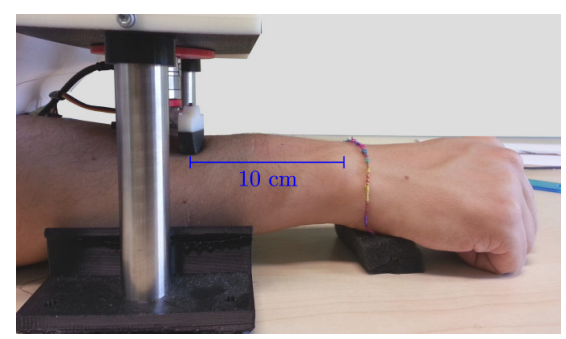

(c) Side view

(10 $\mathrm{cm}$ proximal to the lunate bone)

Fig. 5. Absolute and differential threshold experiments. We considered a modified version of the device, equipped with only one end-effector placed on the dorsal sides of the arm. A six axis ATI Nano 25 sensor was installed between the static platform and an external structure to monitor the interaction forces between the device and the skin. Subjects were required to wear noise-canceling headphones and were blindfolded.

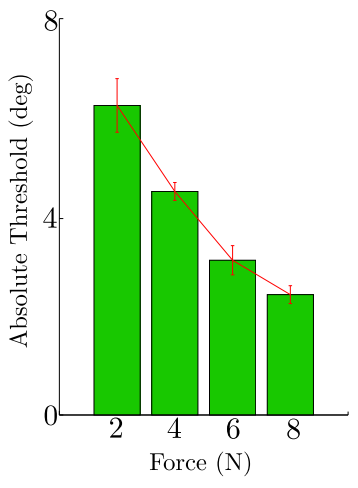

(a) pos. $4 \mathrm{~cm}$

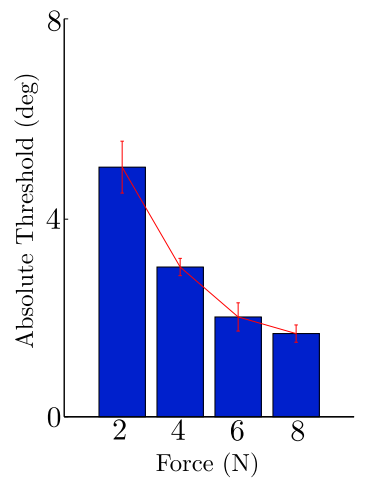

(b) pos. $10 \mathrm{~cm}$
Fig. 6. Absolute thresholds for the eight working conditions. Means and standard deviations are plotted. We tested the metric with the device worn either $4 \mathrm{~cm}$ proximal to the lunate bone (data in (a)) and $10 \mathrm{~cm}$ proximal to the lunate bone (data in (b)). Moreover, we considered the cases of the end-effector exerting a normal force to the skin of $2 \mathrm{~N}, 4 \mathrm{~N}, 6 \mathrm{~N}$, and $8 \mathrm{~N}$.

position further from the wrist provides a better performance (i.e., a lower threshold).

\section{B. Differential threshold}

The differential threshold can be defined as "the smallest amount of stimulus change necessary to achieve some criterion level of performance in a discrimination task" [27]. This gives us information about how different two displacements provided with our device need to be in order to be perceived as different by the human user. This threshold is often referred to as just-noticeable difference or JND. The differential threshold of a perceptual stimulus reflects also the fact that people are usually more sensitive to changes in weak stimuli than they are to similar changes in stronger or more intense stimuli. The German physician Ernst Heinrich Weber proposed the simple proportional law $J N D=k I$, suggesting that the differential threshold increases with increasing intensity $I$ of the stimulus. Constant $k$ is thus referred to as "Weber's fraction".

Schorr et al. [7], in order to evaluate the potential for skin stretch feedback to be used as a sensory substitute for kinesthetic feedback in robotic teleoperation systems, measured the ability of users to discriminate environment stiffness using varying levels of skin stretch at the finger pad. Results showed a mean Weber fractions of 0.168. Similarly,
TABLE I

WEBER FRACTIONS REGISTERED IN THE EIGHT WORKING CONDITIONS FOR EACH REFERENCE STIMULUS.

\begin{tabular}{|c|c|c|c|c|}
\cline { 2 - 5 } \multicolumn{1}{c|}{} & \multicolumn{4}{c|}{ Normal force $f_{n}$ at the skin } \\
\hline standard stimulus & $2 \mathrm{~N}$ & $4 \mathrm{~N}$ & $6 \mathrm{~N}$ & $8 \mathrm{~N}$ \\
\hline $10^{\circ}$ & 0.225 & 0.200 & 0.150 & 0.125 \\
\hline $20^{\circ}$ & 0.137 & 0.125 & 0.112 & 0.087 \\
\hline $30^{\circ}$ & 0.108 & 0.092 & 0.083 & 0.075 \\
\hline
\end{tabular}

(a) $4 \mathrm{~cm}$ proximal to the lunate bone

\begin{tabular}{|c|c|c|c|c|}
\cline { 2 - 5 } \multicolumn{1}{c|}{} & \multicolumn{4}{|c|}{ Normal force $f_{n}$ at the skin } \\
\hline standard stimulus & $2 \mathrm{~N}$ & $4 \mathrm{~N}$ & $6 \mathrm{~N}$ & $8 \mathrm{~N}$ \\
\hline $10^{\circ}$ & 0.150 & 0.125 & 0.100 & 0.075 \\
\hline $20^{\circ}$ & 0.112 & 0.087 & 0.075 & 0.062 \\
\hline $30^{\circ}$ & 0.091 & 0.083 & 0.075 & 0.067 \\
\hline
\end{tabular}

(b) $10 \mathrm{~cm}$ proximal to the lunate bone

Guinan et al. [29] found a mean Weber fraction of 0.2 for their skin stretch sliding plate tactile device.

The experimental setup was the same as described in Sec. III-A. The same ten participants participated also in this experiment. This experiment was carried out right after the one described in Sec. III-A. We evaluated the differential threshold using again the simple up-down method [28]. We used again a step-size $\Delta \alpha=1^{\circ}$, that corresponded to a stretch of $0.35 \mathrm{~mm}$ on the skin. We considered the task completed when six reversals occurred. Subjects were required to wear the cutaneous device as shown in Fig. 5 and tell the experimenter when the two stretches provided felt different. We tested the JND at three standard stimuli: $10^{\circ}, 20^{\circ}$, and $30^{\circ}$, which corresponded to stretches of $3.5 \mathrm{~mm}, 7 \mathrm{~mm}$, and $10.5 \mathrm{~mm}$, respectively. Similarly to Sec. III-A, each participant performed sixteen trials of the simple up-down procedure, with two repetitions for each of the four force values considered ( $2 \mathrm{~N}, 4 \mathrm{~N}, 6 \mathrm{~N}, 8 \mathrm{~N})$ and each of the two position considered $(4 \mathrm{~cm}$ and $10 \mathrm{~cm}$ proximal to the lunate bone). Fig. 7 shows the differential thresholds registered for each reference stimulus in the eight working conditions, while Table I shows the corresponding Weber fractions. By examining the lateral forces registered through the ATI F/T 


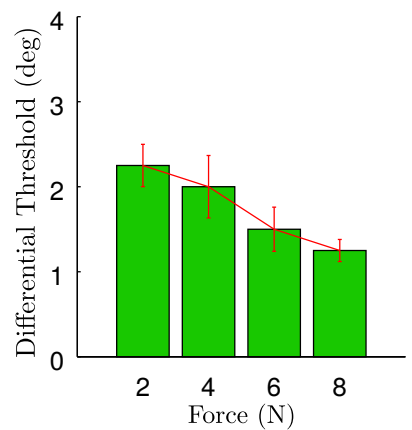

(a) pos. $4 \mathrm{~cm}$, stand. stim. $10^{\circ}$

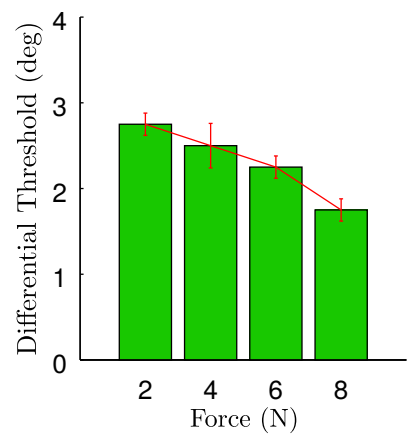

(c) pos. $4 \mathrm{~cm}$, stand. stim. $20^{\circ}$

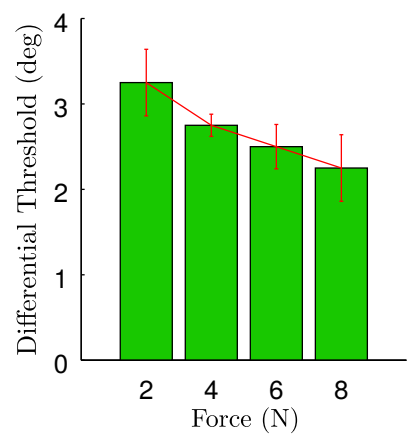

(e) pos. $4 \mathrm{~cm}$, stand. stim. $30^{\circ}$

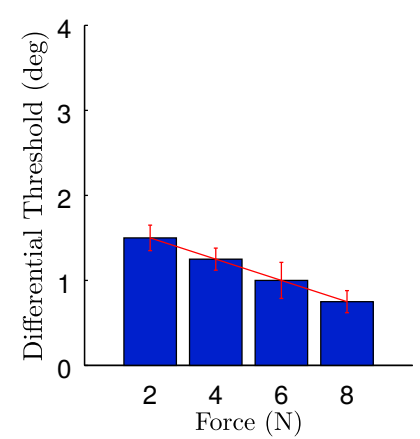

(b) pos. $10 \mathrm{~cm}$, stand. stim. $10^{\circ}$

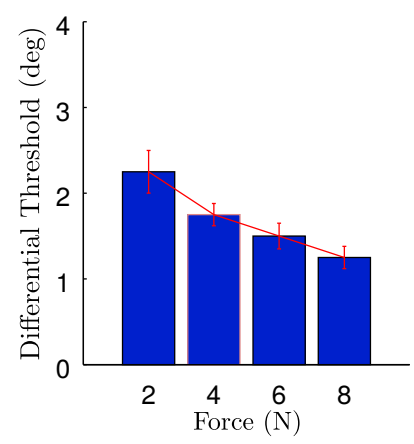

(d) pos. $10 \mathrm{~cm}$, stand. stim. $20^{\circ}$

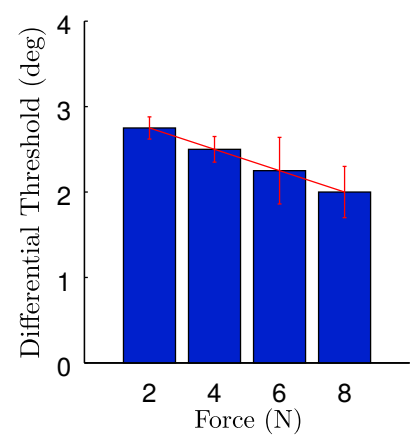

(f) pos. $10 \mathrm{~cm}$, stand. stim. $30^{\circ}$
Fig. 7. Differential thresholds for the eight working conditions and for each reference stimulus considered. Means and standard deviations are plotted.

sensor it was verified that no slippage effect took place during these trials.

Immediately after the experiment, participants were asked to fill in a 5-item questionnaire using bipolar Likert-type seven-point scales. The questions evaluated the comfort of the cutaneous device when tight at the four levels of force considered. A score of 7 described wearing the device as "very comfortable" and a score of 1 as "very uncomfortable". Fig. 8 shows the evaluation of each question. In addition to this questionnaire, subjects were also asked which position of the bracelet they preferred. Seven out of ten preferred when the device was placed $10 \mathrm{~cm}$ proximal to the lunate bone.

\section{Discussion}

As clear from the results reported in Figs. 6 and 7, the highest levels of performance (lower thresholds) were

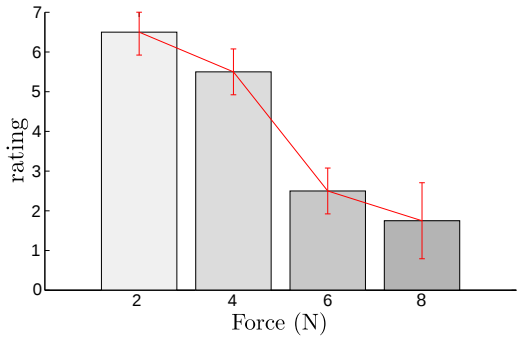

Fig. 8. Comfort level. Participants rated the comfort level at the four different levels of tightness of the device on the arm $(1=$ very uncomfortable, $7=$ very comfortable). Means and standard deviations are plotted.

obtained when the bracelet was tightly fasten to the arm and placed more distant from the wrist. However, we can see from Fig. 8 how fastening the device too tight results in a great discomfort for the user. In order to find a trade-off between performance and comfort, we decided to place the device $10 \mathrm{~cm}$ proximal to the lunate bone and fasten it to the arm until the end-effector applies a force of $4 \mathrm{~N}$ normal to the skin.

\section{EXPERIMENTAL EVALUATION}

To evaluate the effectiveness of our device in providing informative and intuitive shear cutaneous stimuli at the user's arm, we carried out an experiment of haptic navigation. In fact, when all the end-effectors rotate in the same direction, the cutaneous device is able to provide cutaneous cues about a desired pronation/supination of the forearm (see Figs. 3a and $3 \mathrm{~b}$ ), and when two opposite end-effectors rotate in different directions, the cutaneous device is able to provide cutaneous cues about a desired translation of the forearm (see Figs. $3 c$ and $3 d$ ).

The experimental setup was composed of our skin stretch device in its complete four-end-effectors configuration, as shown in Figs. 1 and 2. As discussed in Sec. III-C, we placed the device $10 \mathrm{~cm}$ proximal to the lunate bone and fastened it to the arm until the end-effector applied a force of $4 \mathrm{~N}$ normal to the skin. To easily detect pronation, supination, and translation of the forearm, we used a Leap Motion (http://leapmotion.com) controller, which is a small USB peripheral device composed of two monochromatic IR cameras and three infrared LEDs. It observes a hemispherical area up to a distance of $1 \mathrm{~m}$ with an accuracy up to $0.01 \mathrm{~mm}$ and $0.1^{\circ}$.

The task consisted of rotating or translating the forearm accordingly to the navigation information provided by the device, being as accurate as possible. A video of the experiment can be downloaded at http://goo.gl/LBmhkT. Subjects were required to wear noise-canceling headphones and were blindfolded. Reference rotations and translations were uniformly chosen in the range $\theta_{r} \in(10,50)^{\circ}$ and $d_{r} \in(10,50) \mathrm{mm}$, respectively. The Leap Motion was in charge of registering the position and orientation of the forearm.

\section{A. Subjects and Methods}

Ten participants took part in the experiment, including two women and eight men. Five of them had previous experience 
with haptic interfaces. None of the participants reported any deficiencies in their haptic perception abilities, and all of them were right-hand dominant. Each participant made twentyfour randomized trials of the navigation task, with twelve repetitions for each condition proposed:

- navigation feedback about the desired rotation/translation of the forearm employing our skin stretch device (condition $\mathrm{CF}$ ),

- no cutaneous navigation feedback (condition $\mathrm{N}$ ),

In condition $\mathrm{CF}$, the proposed wearable cutaneous device provides the subject with navigation information about pronation, supination, and translation of the forearm, as described in Sec. II and illustrated in Fig. 3. Subjects are required to rotate/translate the forearm accordingly to the cutaneous information provided by the device. The more the subject rotates/translates the forearm toward the target, the less stretch the device applies to the skin. When the subject reaches the desired orientation/position, the device applies no stretch to the skin. In order to avoid slippage and provide the highest accuracy, the cutaneous device always maps the target rotation/translation to a skin stretch in the range $(-8.75,8.75) \mathrm{mm}$, which corresponds to a range of rotation of the pulleys of $\theta_{p} \in(-25,25)^{\circ}$. This simple proportional control policy for rotations and translation is summarized below.

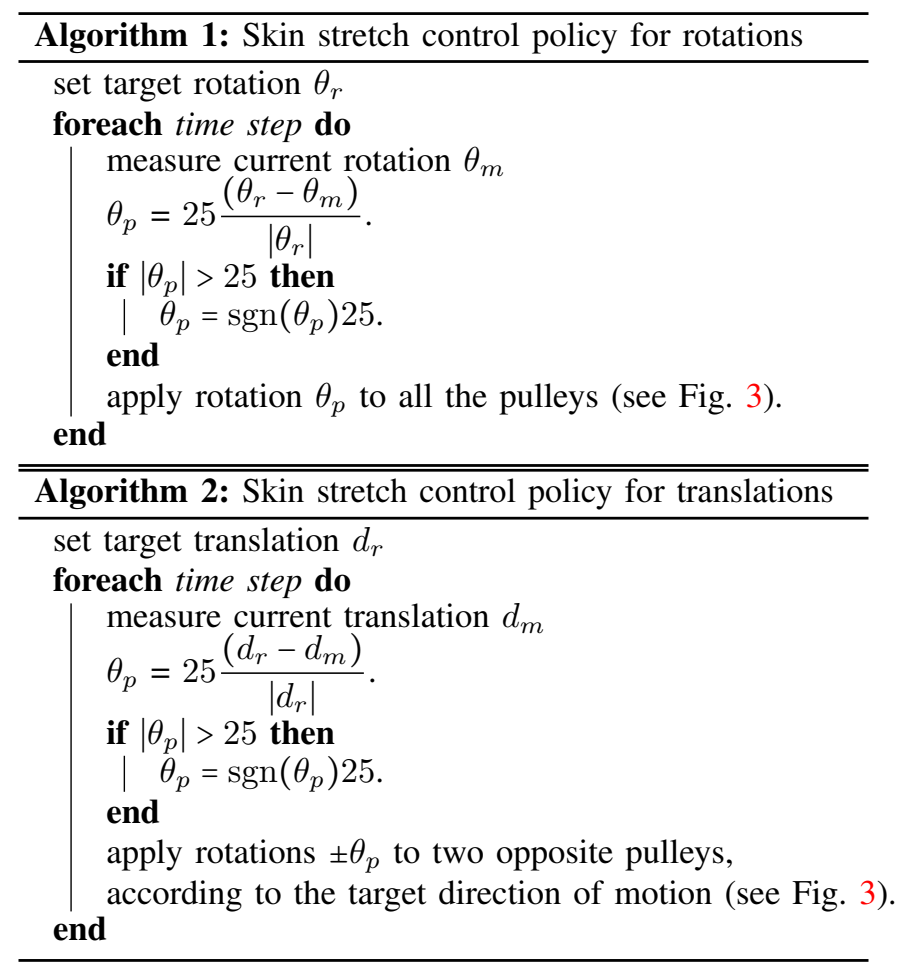

In condition $\mathrm{N}$, no cutaneous feedback is provided to the subjects. The experimenter communicates verbally to the subject the desired movement.

Subjects performed twelve repetitions of the navigation task in each condition, two times for each possible navigation modality: pronation (Fig. 3a), supination (Fig. 3b), translation to the right (Fig. 3d), translation to the left, elevation (Fig. 3c), and lowering of the forearm.

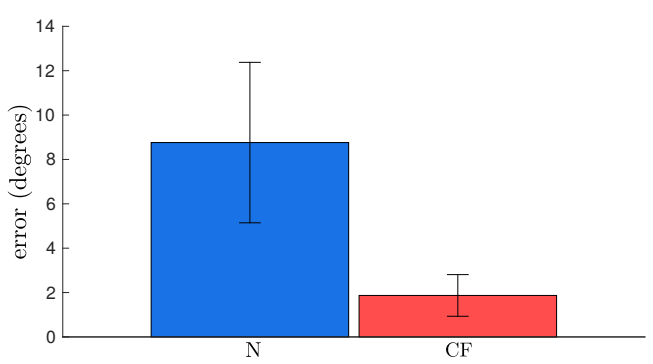

(a) Rotation error

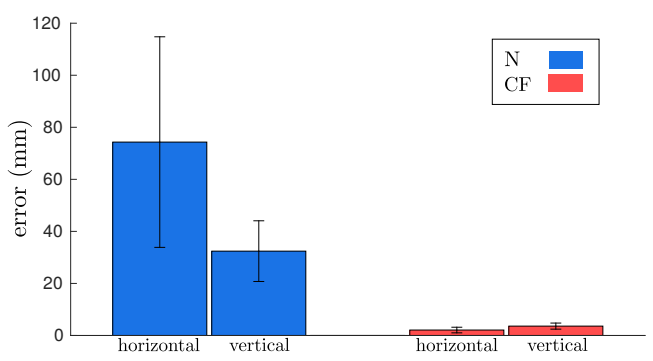

(b) Translation error

Fig. 9. Navigation task. The task consisted in rotating/translating the forearm accordingly to the navigation information provided by the device, being as accurate as possible. Absolute orientation and position error provided a measure of performance. Mean and Standard Error of the Mean (SEM) are plotted.

\section{B. Results and Discussion}

Mean rotation and positioning errors provided a measure of performance. A low value of these metrics denotes the best performance.

Fig. 9a shows the average absolute rotation error at the end of the task. All the data passed the Shapiro-Wilk normality test. A paired-samples t-test determined that the error in following the target rotations differed statistically significantly between the conditions $(\mathrm{t}(9)=3.299, \mathrm{p}=0.009)$. Providing cutaneous haptic feedback enabled the subjects to complete the task with a significantly lower error with respect to not providing any force feedback.

Fig. 9b shows the average absolute translation error at the end of the task for each condition, both for horizontal and vertical movements. All the data passed the ShapiroWilk normality test. Since both the feedback condition (CF and $\mathrm{N}$ ) and the direction of motion (horizontal and vertical) variables have two levels of repeated measures, sphericity was assumed. A two-way repeated-measures ANOVA test revealed statistically significant changes between the feedback conditions $(\mathrm{F}(1,9)=22.917, \mathrm{p}=0.001)$. No statistical difference was found between directions of motion. No significant interaction was found between the variables. To determine whether the difference between horizontal and vertical motion can be considered statistically negligible, we used the two one-sided t-test approach (TOST) [30], [31]. The tests revealed statistical equivalence between the two directions. We can therefore state that providing cutaneous haptic feedback enabled the subjects to complete the task with a significantly lower error with respect to not providing any force feedback, and that the performance is equivalent for horizontal and vertical translations.

Immediately after the experiment, subjects were also asked 
to choose the condition they preferred the most. Condition $\mathrm{CF}$ was preferred by all subjects but one.

\section{CONCLUSIONS}

We presented a cutaneous device able to provide independent skin stretches at the palmar, dorsal, ulnar, and radial sides of the forearm. The device is composed of a bracelet housing four servomotors. Each motor actuates one cylindrical shape end-effector. When in contact with the skin, the four end-effectors are able to generate modulated rotations that can produce controlled stretches at the user's skin. This paper presented the details of the device design and development, together with the results of two experiments. To evaluate how to correctly modulate the reference input of the device, where to locate it, and how to wear it to evoke the most effective cutaneous sensations, we run one experiment to quantify its absolute and differential thresholds. After this, we performed a navigation experiment, in which the device was used to provide navigation information about a desired orientation and position of the user forearm. Results demonstrate an average rotation and translation error of $1.87^{\circ}$ and $2.84 \mathrm{~mm}$, respectively. Moreover, $90 \%$ of the subjects found our device effective in conveying navigation information.

In the near future, we plan to add one vibrotactile motor to the device in order to be able to provide accurate transient/alert sensations. Moreover, we plan to compare our cutaneous device with other devices able to provide navigation information through cutaneous stimuli. Finally, we plan to use the Leap Motion to control the motion of a telemanipulator and then use our cutaneous device to provide the operator with navigation information about environmental obstacles, robot configuration, and active constraints.

\section{REFERENCES}

[1] R. S. Johansson and $\AA$. B. Vallbo, "Tactile sensibility in the human hand: relative and absolute densities of four types of mechanoreceptive units in glabrous skin." The Journal of Physiology, vol. 286, no. 1, pp. 283-300, 1979.

[2] B. B. Edin and N. Johansson, "Skin strain patterns provide kinaesthetic information to the human central nervous system." The Journal of Physiology, vol. 487, no. 1, pp. 243-251, 1995.

[3] K. Minamizawa, S. Fukamachi, H. Kajimoto, N. Kawakami, and S. Tachi, "Gravity grabber: wearable haptic display to present virtual mass sensation," in Proc. ACM Special Interest Group on Computer Graphics and Interactive Techniques Conference, 2007, pp. 8-es.

[4] K. J. Kuchenbecker, D. Ferguson, M. Kutzer, M. Moses, and A. M. Okamura, "The touch thimble: Providing fingertip contact feedback during point-force haptic interaction," in Proc. Symposium on Haptic Interfaces for Virtual Environment and Teleoperator Systems, 2008, pp. 239-246.

[5] D. Prattichizzo, F. Chinello, C. Pacchierotti, and M. Malvezzi, "Towards wearability in fingertip haptics: a 3-dof wearable device for cutaneous force feedback," IEEE Transactions on Haptics, vol. 6, no. 4, pp. 506-516, 2013.

[6] C. Pacchierotti, Cutaneous haptic feedback in robotic teleoperation, ser. Springer Series on Touch and Haptic Systems. Springer International Publishing, 2015.

[7] S. B. Schorr, Z. F. Quek, R. Y. Romano, I. Nisky, W. R. Provancher, and A. M. Okamura, "Sensory substitution via cutaneous skin stretch feedback," in Proc. IEEE International Conference on Robotics and Automation, 2013, pp. 2341-2346.

[8] Z. F. Quek, S. B. Schorr, I. Nisky, A. M. Okamura, and W. R. Provancher, "Sensory augmentation of stiffness using fingerpad skin stretch," in Proc. World Haptics Conference, 2013, pp. 467-472.
[9] C. Pacchierotti, L. Meli, F. Chinello, M. Malvezzi, and D. Prattichizzo, "Cutaneous haptic feedback to ensure the stability of robotic teleoperation systems," International Journal of Robotics Research, vol. 34, no. 14, pp. 1773-1787, 2015.

[10] L. Meli, S. Scheggi, C. Pacchierotti, and D. Prattichizzo, "Wearable haptics and hand tracking via an rgb-d camera for immersive tactile experiences," in Proc. ACM Special Interest Group on Computer Graphics and Interactive Techniques Conference, 2014, p. 56.

[11] D. Prattichizzo, F. Chinello, C. Pacchierotti, and K. Minamizawa, "Remotouch: A system for remote touch experience," in Proc. of IEEE International Symposium on Robots and Human Interactive Communications, 2010, pp. 676-679.

[12] M. Solazzi, A. Frisoli, and M. Bergamasco, "Design of a cutaneous fingertip display for improving haptic exploration of virtual objects," in Proc. IEEE International Symposium on Robots and Human Interactive Communications, 2010, pp. 1-6.

[13] J. B. F. V. Erp, H. A. H. C. V. Veen, C. Jansen, and T. Dobbins, "Waypoint navigation with a vibrotactile waist belt," ACM Transactions on Applied Perception, vol. 2, no. 2, pp. 106-117, 2005.

[14] K. Tsukada and M. Yasumura, "Activebelt: Belt-type wearable tactile display for directional navigation," in UbiComp 2004: Ubiquitous Computing, 2004, pp. 384-399.

[15] H. Kim, C. Seo, J. Lee, J. Ryu, S. Yu, and S. Lee, "Vibrotactile display for driving safety information," in Proc. IEEE Intelligent Transportation Systems Conference, 2006, pp. 573-577.

[16] B. T. Gleeson, S. K. Horschel, and W. R. Provancher, "Design of a fingertip-mounted tactile display with tangential skin displacement feedback," IEEE Transactions on Haptics, vol. 3, no. 4, pp. 297-301, 2010.

[17] R. L. Koslover, B. T. Gleeson, J. T. de Bever, and W. R. Provancher, "Mobile navigation using haptic, audio, and visual direction cues with a handheld test platform," IEEE Transactions on Haptics, vol. 5, no. 1, pp. 33-38, 2012.

[18] I. Oakley, Y. Kim, J. Lee, and J. Ryu, "Determining the feasibility of forearm mounted vibrotactile displays," in Proc. Haptic Interfaces for Virtual Environment and Teleoperator Systems, 2006, pp. 27-34.

[19] A. Spiers, A. Dollar, J. van der Linden, and M. Oshodi, "First validation of the haptic sandwich: a shape changing handheld haptic navigation aid," in Proc. 17th International Conference on Advanced Robotics, 2015, pp. $144-151$.

[20] M. F. Rotella, K. Guerin, X. He, and A. M. Okamura, "Hapi bands: a haptic augmented posture interface," in IEEE Haptics Symposium (HAPTICS), 2012, pp. 163-170.

[21] T. Amemiya and H. Sugiyama, "Orienting kinesthetically: A haptic handheld wayfinder for people with visual impairments," ACM Transactions on Accessible Computing, vol. 3, no. 2, p. 6, 2010.

[22] K. Bark, J. Wheeler, P. Shull, J. Savall, and M. Cutkosky, "Rotational skin stretch feedback: A wearable haptic display for motion," IEEE Transactions on Haptics, vol. 3, no. 3, pp. 166-176, 2010.

[23] T. Nakamura, N. Nishimura, M. Sato, and H. Kajimoto, "Development of a wrist-twisting haptic display using the hanger reflex," in Proc. of the 11th Conference on Advances in Computer Entertainment Technology, 2014 , p. 33

[24] Y. Kuniyasu, M. Sato, S. Fukushima, and H. Kajimoto, "Transmission of forearm motion by tangential deformation of the skin," in Proc. of the 3rd Augmented Human International Conference, 2012, p. 16.

[25] A. Stanley, K. J. Kuchenbecker et al., "Evaluation of tactile feedback methods for wrist rotation guidance," IEEE Transactions on Haptics, vol. 5, no. 3, pp. 240-251, 2012.

[26] V. Yem, M. Otsuki, and H. Kuzuoka, "Development of wearable outercovering haptic display using ball effector for hand motion guidance," in Haptic Interaction, 2015, pp. 85-89.

[27] G. A. Gescheider, Psychophysics: the fundamentals. Psychology Press, 2013.

[28] H. Levitt, "Transformed up-down methods in psychoacoustics," The Journal of the Acoustical Society of America, vol. 49, no. 2B, pp. 467-477, 1971.

[29] A. L. Guinan, M. N. Montandon, A. J. Doxon, and W. R. Provancher, "Discrimination thresholds for communicating rotational inertia and torque using differential skin stretch feedback in virtual environments," in Proc. IEEE Haptics Symposium, 2014, pp. 277-282.

[30] G. B. Limentani, M. C. Ringo, F. Ye, M. L. Bergquist, and E. O. McSorley, "Beyond the t-test: statistical equivalence testing," Analytical chemistry, vol. 77, no. 11, pp. 221-226, 2005.

[31] C. Pacchierotti, D. Prattichizzo, and K. J. Kuchenbecker, "Displaying sensed tactile cues with a fingertip haptic device," IEEE Transactions on Haptics, vol. 8, no. 4, pp. 384-396, 2015. 